\title{
Ueber das Nltramarin;
}

\section{von Dr. Carl Böttinger.}

(Eingelanfen den 28. April 1876.)

Im Laufe des verflossenen Sommers hatte ich Gelegenheit, mich etwas näher mit dem Studium des Ultramarins zu beschäftigen und glaube ich die Ergebnisse der Untersuchung um so weniger der wissenschaftlichen Welt vorenthalten $\mathbf{z u}$ dürfen, als mich die Art und Weise des Verlaufs derselben zu der Annahme berechtigt, dafs der eingeschlagene Weg leichter wie jeder andere zuın Ziele führen mag. Gleichwohl ist es mir selbst noch nicht gelungen, dieses zu erreichen.

Naturgernäfs zerfällt eine derartige Arbeit in mehrere Abschnitte. Ich werde mich bemühen, im Nachfolgenden das umfangreiche Material so zu ordnen, dafs es bei möglichstem Zusammenfassen das Wesen der Sache klar hervortreten läfst.

Bekanntlich werden zur Bildung des Ultrumarins folgende Körper verwendet : Thon, kohlensaures Natrium (Sulfat), Schwefel und Kohle. Häufig wird dem Thone noch eine gewisse Menge freier Kieselsäure beigefügt, um dem zu erzielenden Product besondere Eigenschaften zu ertheilen.

Indem ich nun von einem Satze ausging, dessen Zusammensetzung ich kannte und welcher beim Brennen eine schöne blaue Waare lieferte, suchte ich den Einflufs einer Variation der Mengen der einzelnen Componenten desselben auf das Aussehen, die Natur und in der Analyse des erzielten Productes zu ergründen.

Zunächst studirte ich den Einflufs des Verhältnisses zwischen Kieselsāure und Thonerde bei gleichbleibender oder nur wenig schwankender Schwefel- und Sodamenge. Das 
Verhältnifs dieser beiden Bestandtheile im Satze war in folgenden drei Fällen :

$$
\frac{\mathrm{SiO}_{2}}{\mathrm{Al}_{2} \mathrm{O}_{8}}=\begin{array}{ccc}
1 . & 2 . & 3 . \\
2,9 & 2,9 & 2,57 .
\end{array}
$$

Die Analysen der daraus erzielten Rohbrande *) ergaben folgende Zahlen :

1.

$\begin{array}{lr}\mathrm{SiO}_{3} & 39,70 \\ \mathrm{Al}_{2} \mathrm{O}_{3} & 25,58 \\ \mathrm{CaO} & 0,31 \\ \mathrm{~S} & 13,07 \\ \mathrm{Na}_{9} \mathrm{O} & 21,71\end{array}$

2.

38,75

25,44

0,30

13,01

21,76
3.

39,07

26,04

0,77

$: 2,93$

21,52 .

Die Verhältnisse $\frac{\mathrm{SiO}_{2}}{\mathrm{Al}_{2} \mathrm{O}_{3}}$ sind demnach :

1.

2,67
2.

2,7
3.

2,56 ,

wie man sieht, obigen so weit entsprechend, als man bei derartigen Versuchen nur immer erwarten darf.

Um den Einflul's der Kiesclsäure im Satze kennen zu lernen, fügte ich dem bekannten Thone bei constantem Gewichte Soda und Schwefel, mit je fünfpfündiger Steigerung freie Kieselsäure zu. Bezeichne ich den ursprünglichen Satz mit A, so wird die Zusammensetzung der folgenden fünf Sätze ausgedrückt durch die Formen :

4.

5.

$\mathrm{A}+5 \mathrm{SiO}_{2} \mathrm{~A}+10 \mathrm{SiO}_{2}$

Die Analysen der aus diesen Compositionen gewonnenen Ultramarine ergaben :

4.

$\mathrm{BiO}_{8} \quad 39,35$

$\mathrm{Al}_{\mathbf{2}} \mathrm{O}_{\mathbf{2}}$

$\mathbf{8}$
5.

39,35

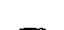

6.

$A+15 \mathrm{SiO}_{2}$
7.

$\mathrm{A}+20 \mathrm{SiO}_{8}$
8.

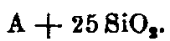


Das Aussehen der Proben 4, 5, 6 war normal, in der Mitte der Tiegel 7 und 8 befanden sich jedoch grölsere geschinolzene Kerne.

(Ich bemerke hier, dafs alle Proben doppelt und in verschiedenen Oefen gebrannt wurden. Aufserdem dafs, wie es Hoffmann schon gefunden, der Kieselsäure häufig sogenemnter Thonrückstand beigemengt ist, ich jedoch diesen, da er zumeist aus $\mathrm{SiO}$, besteht, wenn nichts anders bemerkt, zo derselben gercelinet hahe.)

Der Bildung dieser Kerne ist jedoch in den meisten Fällen vorzubeugen, wenn man die Schwefelmenge des Satzes vermehrt. Als ich den Sätzen 7 und 8 (einige Centner) mehr Schwefel beifügte, erhielt ich nach dem Brennen schōnes röthlich-blaues Uitramarin. Die Analysen dieser Producte ergaben :

$\begin{array}{lrr} & 7 \mathrm{~b} . & 8 \mathrm{~b} . \\ \mathrm{SiO}_{8} & 44,41 & 44,92 \\ \mathrm{Al}_{2} \mathrm{O}_{8} & 25,88 & 24,71 \\ \mathrm{~S} & 13,55 & 12,96 .\end{array}$

Der Kieselsäure sind bei $7 \mathrm{~b} 2,5 \mathrm{pC}$, bei $8 \mathrm{~b} 3,9 \mathrm{pC}$. Thonrïckstand zugerechnet.

Diest Zahlen zeigen in Algemeinen, dafs das Ultramarin um so reicher an Säure ist, je kieselsäurereicher der Satz.

Indem ich von der besonderen Wirkung der Kieselsäure beim Brennen des Satzes absebe, kann ich nicht umhin, daran zu erinnern, dafs es einen bedeutenden Unterschied im Ausfallen des Productes bedingt, ob die Säure in chernischer Verbindung mit Thonerdt, als Thon, oder ob sie als solche frei zugeschlagen und wenn diefs der Fall, ob sie amorphe oder krystallisirte Säure ist. Bekanntlich werden bei Anreicherung des Satzes mit Kieselsäure die erzeugten Ultramarine säurebeständiger. Es mag daher nicht uninteressant erscheinen, folgendea Versuch hier einzuschalten. 
Rührt man Ultramarin mit schwach alkalisch reagirendem Wasserglas zu einem dicken Brei an, trocknet die Masse, erhitzl sie dann auf eine Temperatur von circn $200^{\circ}$, laugt sie endlich mit etwas Wasser aus, so findet man bei vergleichenden Versuchen, dafs das erbaltene Product sãurebeständiger geworden ist.

Obgleich sich dieses Verfahren nicht zur technischen Herstellung des Products eignet, so bietet es doch einen Fingerzeig auf den Vorgang im Ofenprocesse.

Aus dem Vorhergehenden ergiebt sich, dafs zur Bildung eines guten Ultramarins ein constantes Verhältnifs von $\mathrm{SiO}_{2}$ : $\mathrm{Al}_{2} \mathrm{O}_{3}$ nicht erforderlich ist, vorausgesetzt, dafs Sorge getragen wird, ein bestimmtes Verhältnifs zwischen $\mathrm{SiO}_{2}$ und $\mathrm{S}$ nicht zu unterschreiten.

Ich bereitete mir daher bei weiteren Versuchen, welche den Einflurs der Soda urd des Schwefels erkennen lassen sollten, einen Thon tnit constantem Verhältnils $\frac{\mathrm{SiO}_{8}}{\mathrm{Al}_{2} \mathrm{O}_{3}}$. Dasselbe war $2,5: 1$. Hundert Theilen dieses Thons wurden nachverzeichnete Merigen Soda und Schwefel zugefügt. Ich lege hier kein Gewicht darauf, das Aussehen der betreffenden Brände anzuführen, weil ich nachher Gelegenheit nehmen werde, dieses nachzuholen.

Zusammenselsung der Sä̌se :

\begin{tabular}{|c|c|c|c|}
\hline 9. $21 / 2 \mathrm{BiO}_{\mathrm{a}}: 1 \mathrm{Al}_{8} \mathrm{O}_{\mathrm{a}}$ & \multicolumn{2}{|c|}{$45 \mathrm{Na}_{2} \mathrm{CO}_{8}$} & 308 \\
\hline 10. & 50 & $n$ & $35 n$ \\
\hline 11. & 55 & & 40, \\
\hline 12. & 60 & $n$ & $45 n$ \\
\hline 18. & 65 & $n$ & 50, \\
\hline 14. & 70 & $n$ & 55, \\
\hline 15. & 70 & $n$ & 70 \\
\hline 16. & 70 & $n$ & 80, \\
\hline 17. & 90 & $\bullet$ & 80 , \\
\hline 18. & 100 & r & 90, \\
\hline 19. & 65 & $\bullet$ & 80 ה \\
\hline
\end{tabular}




\section{Analysen dor Rohbrande :}

$\begin{array}{rrlc} & \mathrm{BiO} & \mathrm{Al}_{8} \mathrm{O}_{8} & \mathrm{8} \\ \text { 9. } & 53,96 & 25,61 & 6,74 \\ 10 . & 62,32 & 27,00 & 9,38 \\ 11 . & 47,38 & 27,43 & 4,20 \\ 12 . & 46,66 & 26,37 & 9,65 \\ 13 . & 47,00 & 27,92 & 8,63 \\ 14 . & 44,60 & 30,08 ? & 8,66 \\ 16 . & 42,54 & 26,15 & 10,86 \\ 16 . & 41,84 & 24,25 & 12,77 \\ 17 . & 45,87 & - & 10,98 \\ 18 . & 42,68 & 26,66 & 14,80 \\ 19 . & 61,83 & 20,10 & 10,77 .\end{array}$

Von diesen empirisch gewählten Sätzen ging ich über zu solchen, in welchen auch der Schwefel und das Natrium der Soda in einem bestimmten atomistischen Verhältnisse gewählt worden waren.

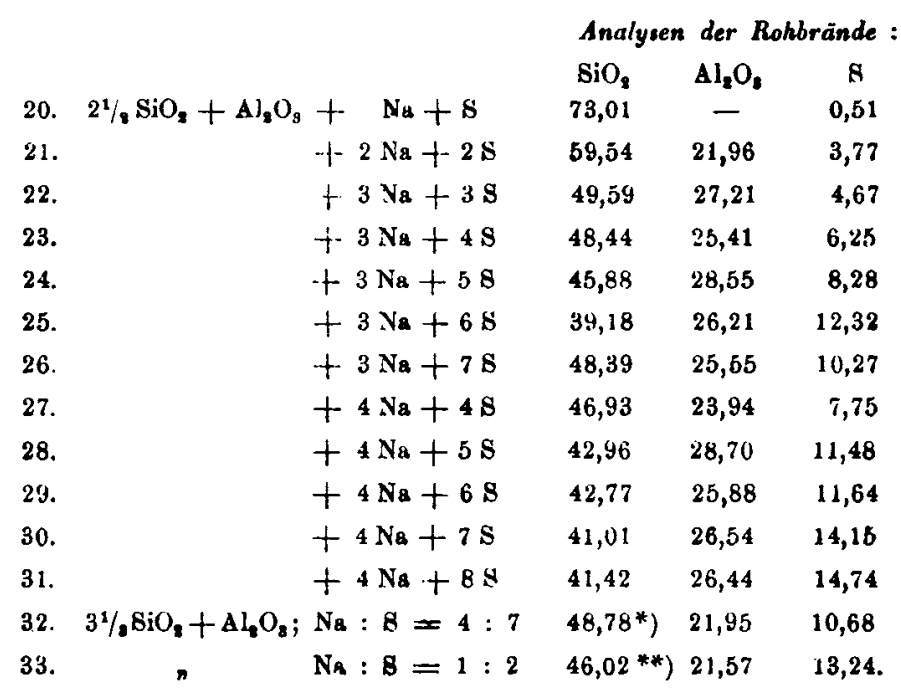

*) Der Kieselekure sind 6,92 pC. 'Thonrickstand beigeschrieben.

**) Der Kieselesure sind 5,11 pC. Thonrüakstand beigeschrieben. 
In Folgenden führe ich in Kürze die Eigenschaften der Proben 20 bis 33 an.

20. Röthlichweifse, lockere, zusammengeballtr Masse.

21. Graugelbe, homogene Magse.

22. Graugelbe Masse, mit grünen Purkten durchsetzt.

23. Vom Tiegelrande nach Innen blau, im Inneren lockere grüne Masse mit vielen blauen Punkten.

24. (Tiegel gesprungenj. Weifser Rand, grüne, etwas gelbe, wenig blane Masse.

25. Obere Masse trübes Blan, sonst total geschmolzen. (Bei Versuchen im Grofsen geschah diefs nicht.)

26. Am Tiegeirande röthlichblau, Innen grün.

27. Graugelbe Masse mit grünlichen Einsprengangen.

28. Harte, schwere Masse, blaue, aber trïbe Farbe.

29. Harte, klingende Masse, durchaus grüne Farbe.

30. Blaue Farbe.

31. Jchöno blaue Farbe.

82. Grünliches Blau, entbalt farblose Krystallfragmente, wolche sich schun in Thone vorfanden.

33. Schünes Blau mit röthlichem Stich. (Enthält gleichfalls Krystallchen:

Diese Angaben zeigen aufs Unzweideutigste, dafs dem Thone hei seiner Vereinigung mit $\mathrm{Na}_{q} \mathrm{O}$, resp. dem so entstandenen Thonerdenatronsilicat die Fähigkeit zukommt, sich mit irgend welchen Schwefelverbindungen zu vereinigen, und dafs das Iltramarin nur das Endglied ciner grofsen Reihe derartiger Körper ist, mit dor ausgesprochenen Eigenschaft, von Sauren vollkommen zerlegt zu werden, wie diefs von Hoffmann früherhin gleichialls, wenn auch in etwas anderem Sinne uusgesprochen wurde. Die Zusammensetzung dieses Silicats zu ermittrin ist mir trotz vieler Berechnungen nicht gelungtn. Doch scheint es mir zum wenigsten zweifelhaft, dafs der Schwefel in directer Verbindung mit dem Silicium stehe, da es mir ungrachtet zahlreicher Versuche nicht gelungan ist, krystallisirtes Silicium in eine Schwefelverbindung umzuwandeln. Ich hatte vielmehr für wahrscheinlicher, dafs 
wir es hier nicht mit einer atomistischen, sondern nach $\mathbf{A r t}$ der Doppelsalze mit einer Molecularverbindung zu thun haben. Nur durch Analyse die Zusarnmensetzung des blanen Farbstoffs ergründen zu wollen, scheint mir aufserdem schon deshalb schwer, weil der verwendete Thon ein Gemenge aller möglichen Silicate sein kann und es bei aller Sorgfalt schwierig ist, starre Körper so zu mischen, dafs sie sich in dem Tiegelraum gleich vertheilt finden. Aufserdem zeigt die Farbe, je nach dem Orte, von welchem man sie entnimmt, eine verschiedene Zusammensetzung.

\section{Zersetzungen des Ultramarins.}

Es ist schon lange bekannt, dafs man häufig die blaue Farbe mit einem weifsen Kranz umgeben findet, namentlich dann, wenn die Mischung in gerissenen Tiegeln erhitzt wurde odei bei deren Brand Tiegelrisse entstanden. Die weifse Masse erstreckt sich vom Risse des Tiegels nach beiden Seiten und nach dem Inneren hin, in den meisten Fällen jedoch nicht sehr weit und bald beginnen blaue Punkte mit zu unterlaufen. Beim Auswaschen von gutern Rohbrand mit Wasser geht bekanntlich Natriumsulfat in Lösung, dessen Menge je nach der Zusammensetzung des Satzes und der Art des Brennens variirt. Wahrend aber gutes Ultramarin circa 13 bis $14,5 \mathrm{pC}$. Schwefel in irgend welcher Form gebunden hält, geben diese Producte fast den ganzen Schwefel in Form von Sulfat an Wasser ab. Die Analyse eines solchen Körpers, welcher einem schön blauen Tiegel entnommen war, ergab nach geeigneter Vorbereitung :

34.

$\begin{array}{lr}\mathrm{SiO}_{2} & 51,63 \\ \mathrm{Al}_{2} \mathrm{O}_{2} & 30,92 \\ \mathrm{~S} & 1,40 .\end{array}$

Die Entstehung dieser weifsen Producte, also die Zerstörung der Farbe und Ueberführung der Schwefelverbindungen 
in auswaschbares Sulfat, sind dem Einflusse des Sauerstoffe zuzuschreiben, wit auch sonst allgemein angenommen wird.

Im Inneren der Tiegel findet man häufig bei schlecht gewählten Verhältnissen des Satzes geschmolzene Massen, welche an die Luft gebracht Feuchtigkeit anziehen und lebhaft $\mathrm{H}_{8} \mathrm{~S}$ entwickeln. Laugt man ein derartiges Stück mit Wasser aus, so beobachtet man ein höchst eigenthümliches Verhalten. In die kalte wässerige Lösung gehen neben $\mathrm{Na}_{2} \mathrm{SO}_{4}$, $\mathrm{Na}_{8} \mathrm{SO}_{3}, \mathrm{Na}_{8} \mathrm{~S}_{2} \mathrm{O}_{3}$ noch die Natriumsulfide, aber nicht auf einmal, sondern nur bei häufiger Erneuerung des Wassers und um so reichlicher, in je vertheilterem Zustande sie sich befinden. Kocht man ein solches Pulver mit Wasser, so entweicht selbst noch nach Stunden und Tagen $\mathrm{H}_{2} \mathrm{~S}$, dabei gehl die Farbe aus tiefem Schwarz in ein helles Grünlichweifs über.

Ein derartiges Stück wurde gepulvert und mindestens 30 mal mit heifsem und kaltem Wasser ausgelaugt, dann drei Tage in einem mit Kühler verbundenen Kolben gekocht, wobei fortwährend $\mathrm{H}_{\mathbf{y}} \mathrm{S}$ entwich. Das Product wurde hell Grünlichgelb. In der wässerigen Lösung waren Schwefelverbindungen des Natriums gelöst, aufserdem eine bedeutende Menge eines weifslichen Silicats, sowio etwas Schwefel suspendirt. Die wässerige Lösung der Natriumverbindungen wurde durch Filtration von dem Rückstand und den suspendirten Theilen getrennt und verhielt sich gegenüber Reagentien wie folgt :

Reagirt stark alkalisch.

Beim Versetzen eines kleinen Theils der Flüssigkeit mit $\mathrm{HCl}$ entweicht vornehmlich $\mathrm{SO}_{2}$ und machen sich kleine Mengen $\mathrm{H}_{\mathbf{s}} \mathrm{S}$ bemerklich.

Versetzt man etwas des Filtrats mit $\mathrm{BaCl}_{2}$, so entsteht ein Niederschlag, welcher sich nach dem Auswaschen mit Wasser als ein Gemenge von $\mathrm{BaSO}_{4}$ und $\mathrm{BaSO}_{2}$ ausweist. 
Das von demselben getrennte Filtrut zeigte Gehalt von $\mathrm{BaS}_{2} \mathrm{O}_{y}, \mathrm{BaS}$ und $\mathrm{Ba}(\mathrm{OH})_{z}$.

Bei Bebandlung eines Theils der Flüssigkeit mil neutraler, ammoniakalischer und saurer Höllensteinlösung konnte die Gegenwart von $\mathrm{Na}_{2} \mathrm{~S}_{8} \mathrm{O}_{3}, \mathrm{Na}_{8} \mathrm{SO}_{3}$ und $\mathrm{NaSH}$ nachgewiesen werden.

Die Gegenwart von NaHS wurde ferner durch das Verbalten gegen Bleiacetat fusigestellt.

Das ursprünglich $7,2 \mathrm{pC}$. Schwefel haltende gewaschene Product enthielt nach dreitagigem Kuchen nur noch 2,3 pC. Schwefel.

Ein gelbgrünes nicht geschmolzenes Product zeigte beim Kochen mit $W$ asser ein dem eben beschriebenen ganz analoges Verialten. Dasselbe wurd: unausgewaschen verwendet, da es an Wasser nur $\mathrm{Na}_{2} \mathrm{SO}_{4}$ algab.

Fs enthielt unauggewaschen Schwofel . . . $11,79 \mathrm{pC}$.

Auswaschbarer Schwefel (als $\mathrm{Na}_{8} \mathrm{SO}_{4}$ ) . . $2,70 \mathrm{pC}$.

Demnach wirklicher Gohalt an Bchwefel . . 9,09 pC.

Nach einer fünftägigen Kochperiode wurde die Flüssigkeit mit den darin suspendirten Theilen durch Filtration vom Bodensatze getrennt.

Der bei $100^{\circ}$ getrocknete, sehr helle Bodensatz enthielt 8,86 pC. Schwefel.

Das durch Abdampfon gewonnene, bei dieser Operation wieder dunkler werdende, vorher hellgraue, suspendirte Siliost 4,22 pC. Bohwofel.

Auch in diesem Falle bemerkt man eine bedeutende $\mathbf{A b}-$ nahme im Gehalte des gebundenen Schwefels. In der Flüssigkeit finden sich bei alkalischer Reaction die oben beschriebenen Schwefelverbindungen.

Kocht man Ultramarin (beste Waare) mit Wasser am Rūckflufskühler, so beobachtet man dieselben Erscheinungen, allerdings in bedeutend geringerem Grade. So nimmt man die alkalische Reaction der Flüssigkeit erst bei starker Con- 
centration wabr, doch sind dit beschriebenen Schwefelverbindungen auch hier nachweisbar. Desgleichen gelingt es leicht, das langsame, aber stete Entweichen von $\mathrm{H}_{2} \mathrm{~S}$ nachzuweisen.

Ein Ultramarin enthielt unausgowachen Bahwefol - 17,87 pC.

Als $\mathrm{Na}_{2} \mathrm{SO}_{4}$ waren auswachbar Schwefel . . . . 3,46 pC.

Danach gebundener Bohwefel . . . . . . . 14,41 pC.

Nach einer einwöchentlichen Kochperiode, bei welcher täglich das Blauwasser entfernt und reines destillirtes Wasser genommen wurde, enthielt

der bei $100^{\circ}$ getrocknete Bodensatz 13,97 pC. Bchwefel;

der in der Flüssigkeit suspendirt gewesene und durch Abdarnpen gewonnene Theil nach dem Waschen und Trocknen bei $100^{\circ}$ $13,58 \mathrm{pC}$. Schwefol.

Auch das Uitramarin erleidet also bei dieser Operation einen, wenn auch kleiren, Verlust an chemisch gebundenem Schwefel. Es nimmt dabei einen Stich ins Dunkelgrüne an. Ich bemerke, dafs ich diesen Versuch mit zahlreichen Ultramarinsorten angestellt habe und immer dasselbe Verhalten, wenn es auch gradweise verschieden war, constatiren konnte.

Die beschriebenen Zersetzungen erfolgen um so rascher, wenn man Sorge trägt, das Product von Zeit zu Zeit zu zerreiben oder mit frischem Wasser zu übergiefsen. Was das letztere anlangt, so glaube ich, dafs Folgendes geeignet sein dürfte, den Grund dieses Verhaltens erkennen zu lassen.

In die wässerige Lōsung gehen einige Verbindungen, welche sich theilweise mit dem $W$ asser zersetzen (Schwefelalkalien z. B. in NaHO, NaHS und S) und deren Umwandlungsproducte zur Bildung neuer Körper $\mathrm{CNa}_{2} \mathrm{SO}_{4}, \mathrm{Na}_{8} \mathrm{SO}_{8}$, $\mathrm{Na}_{8} \mathrm{~S}_{2} \mathrm{O}_{3}, \mathrm{Na}_{2} \mathrm{~S}$ und $\mathrm{Na}_{8} \mathrm{~S}_{5}$ ) Veranlassung geben kōnnen. Das Silicat, resp. das Ultramarin könnte befähigt sein, sich unter gewissen Umständen nit cinen Theil dieser Producte zu vereinigen, wodurch gewissermafsen ein Gleichgewichtszustand 
zwischen Zersetzung und Nea- resp. Umbildung eintreten würde. Diese Vorstellung scheint mir durch das Folgende noch wahrscheinlicher gemacht $2 u$ werden.

Eine von mir aus Blau künstlich dargestelite grüne Farbe zersetzte sich mit Wasser ganz in der oben geschilderten Weise. Während des Kochens mischte ich demselben etwas Scliwefel zu, wodurch jedoch keine besonders autfallende Erscheinung hervorgerufen wurde. Als ich aber dann ein winziges Stückchen Aetznatron in die siedende Flüssigkeit warf, da verwandelte sich sehr rasch sämmtliches Grün in blaue Farbe. Die überstehende Flüssigkeit zeigte für kurze Zeit eine schmutzig-grüne Parbe, wurde jedoch bei weiterem Kochen bald wieder farblos und verlief alsdann die Reaction in der früheren Weise.

Kocht man Ultramarin mit Wasser, dem man wenige Tropfen $\mathrm{AgNO}_{3}$ zufügt, so entsteht $\mathrm{AggS}_{8}$.

Setzt man den in gleichen Bedingungen befindlichen Ultramarin etwas metallisches Quecksilber zu, so entsteht HoS. Nebenbei entweicht $\mathrm{H}_{\mathbf{y}} \mathrm{S}$, was andeutet, dafs die Lersitzung auch aufserhalb der Wirkungssphäre des $\mathrm{Hg}$ von Statten geht.

Reibt man Ultramarin mit $\mathrm{Hg}$ in einem Achatmörser innig zusammen, so wird HgS gebildet, die Farbe zerstört.

Reibt man Ultranarin mit basischem Bleiacetat, beide in vollkommen trockenem Zustande, innigst zusammen, so entsteht $\mathrm{PbS}$, dabei grht die Farbe zu Grunde.

Reibt man rines trockenes Ultramarin mit ueutralem truckenen $\mathrm{AgNO}_{3}$ innig zusammen, so findet Umselzung statt; die Farbe erleidet dabei die frühere Veränderung.

Reitt man reilles mit $W$ asser benetztes Ultramarin heftig und andeuernd in einem Möser, bringt alsdann elwas davon auf eine geglättele. Visitenkarte $(\mathrm{Pb})$, spült diese mit Wasser $a b$, so entsteht an der Stelle, auf welcher das Ultramarin 
lagerte, ein schwarzer Fleck von PbS. Selbstverständlich zeigt nicht zerriebenes Ultramarin kein derartiges Verhalten.

Reibt man reines trockenes Ultramarin innigst, so zerstört man die Farbe unter dem Strich. (Man nelime nur wenig zu diesem Versuch.) Befeuchtet man die Masse mit Wasser, bringt sie auf ein Saugfilter, wascht etwas nach und engt das Filtrat ein, so zeigt dasselbe starke alkalische Reaction.

Erhitzt man gutes Ultramarin längere Zeit auf $250^{\circ}$, bis man Sicherheit hat, dafs der grörste Theil des $W$ assers und sämmtlicher etwa vorhandene freie Schwefel entwichen ist, so beobachtet man ein Dunkler- und Trüberwerden der Farbe.

Giüht man derartig zubereitetes Ultramarin in einer Kugel- oder Proberöhre, welche geschlossen und durch eine Glasröhrenleitung in Verbindung mit Höllensteinlösung gebracht ist, so bemerkt man Anfangs das Entweichen von $\mathrm{SO}_{\boldsymbol{q}}$, späterhin sublimirt viel freier Schwefel. Diese Erscheinung findet langsam, aber lang andauernd stalt. Wiederholt unter Luftabschlufs geglühtes und wieder erkaltetes Ultramarin zeigte immer von Neuem dasselbe Verhalten.

Erhitzt man dagegen lufttrockencs reines Ultramarin in einem gleichen Apparat, so entweicht anfänglich viel $W$ asser. Treibt man dieses, resp. seinen Dampf in Höllensteinlösung, so beobachtet man neben Reduction einen Niederschlag von $A_{g} S$. Es entweicht daher mit dem $W$ asser noch $H_{8} S$ und es steht dieses gemeinsame Auftreten in einem innigen $\mathrm{Zu}$ sammenhang.

Un die Menge des beim Glühen weggehenden Schwefels zu bestimmen, wiederholte ich den Versuch in quantitativer Weise.

$\mathrm{Zu}$ dieser und allen naciffolgenden Operationen verwendete ich einen schönen blauen Kohbrand in unausgewaschenem 
Zustend. Derselbe gab an $\mathbf{H}_{2} \mathrm{O}$ Sulfat ab und beläuft sich dessen Menge

in Schwefel ausgedrückt anf $3,88 \mathrm{pC}$.

Der gewaschene bei $100^{\circ}$ getrocknete Rohbrand ergab bei der Analyse folgende Zahlen :

$\begin{array}{lc} & 35 . \\ \mathrm{SiO}_{2} & \$ 7,18 \\ \mathrm{Al}_{8} \mathrm{O}_{8} & 22,63 \\ \mathrm{~S} & 12,49 .\end{array}$

Nach den Glühen des unausgewaschenen Rohbrandes waren auswaschbar, $\mathrm{Na}_{8} \mathrm{SO}_{4}$,

in Schwelel ausgedriickt $3,96 \mathrm{pC}$.

Die Analyse des gewaschenen und bei $100^{\circ}$ getrockneten Körpers gab

$35 \mathrm{~b}$.

$\begin{array}{ll}\mathrm{SiO}_{2} & 48,07 \\ \mathrm{Al}_{2} \mathrm{O}_{8} & 22,94 \\ \mathrm{~S} & 11,90 .\end{array}$

Mithin hatte der Rohbrand hei einfachem Glühen 0,51 pC. Schwefel in Form von Gasen und Dämplèn verloren.

Erhitzt man denselben ungewaschenen Rohbrand mit etwa 5 bis $6 \mathrm{pC}$. Kohle während 15 bis 20 Minuten, so entweichen bei niederer Temperatur zunächst $\mathrm{H}_{2} \mathrm{O}$ und $\mathrm{H}_{2} \mathrm{~S}$, etwas $\mathrm{SO}_{z}$ und $\mathrm{S}$, bei hoher Temperatur jedoch $\mathrm{SO}_{8}$, viel $\mathrm{CO}_{8}, \mathrm{CO}$, $\operatorname{COS} *$ ) und S. Schwefelkohlenstoff gelang mir nicht nachzuweisen. Die Farbe des Products wurde nicht allein dunkel, trübe, sondern auch grün. Beim $\Lambda$ uswaschen verlor dasselbe Sulfat, dessen Menge

$$
\text { in Schwefol aungedrückt } 2,85 \mathrm{pC} \text {. }
$$

Die Analyse des gewaschenen, bei $100^{\circ}$ getrockneten Products ergab :

$\begin{array}{ll}\mathrm{SiO}_{2} & 52,29 \\ \mathrm{Al}_{\mathbf{2}} \mathrm{O}_{2} & 19,62 \\ \mathbf{S} & 11,72 .\end{array}$

*) Welcher Körper deber wohl aucb im Ofen auftreten möcbte. 
Der Gesanumtverlust an Schwefel stellt sich bei diesent Versuch auf 1,82 pC., wovon jedenfalls mehr als die Hälfte dem Ultramarin zuzuschreiben ist. Auffallend ist noch die Vermehrung des Thonrückstands.

Erhitzt man Ultramarin mit Soda oder mit Soda und Kohle, so gelingt es, grüne Körper zu erhalten, welche vollkornmen analog jenen sind, die in Ofenprocesse bei schlecht gewähltem Satze gebildet werden, d. h. dann, wenn die Verhältnisse zwischen $\mathrm{SiO}_{2}: \mathrm{Na}$ einestheils, $\mathrm{Na}: \mathrm{S}$ anderntheils ungünstig gewählt sind.

Da ich den Einflufs dieses Verfahrens quantitativ verfolgen wollte, verwendete ich bei nachfolgenden Versuchen das unter 35. analysirte Ultramarin.

1) Erhitzen des Rohbrandes mit $20 \mathrm{pC} . \mathrm{Na}_{2} \mathrm{CO}_{9}$. Das erhaltene Product besitzt eine hellgrüne Farbe. In dem beim Auswaschen erhaltenen Filtrat wurde besonders $\mathrm{Na}_{2} \mathrm{~S}$ nachgewiesen.

Analyse des gewaschenen und bei $100^{\circ}$ getrockneten Körpers :

$\begin{array}{lr}\mathrm{SiO}_{2} & 44,00 \\ \mathrm{Al}_{8} \mathrm{O}_{3} & 20,06 \\ \mathrm{~S} & 8,06 .\end{array}$

Derselbe wurde durch Abbrennen mit Schwefel gebläut und der Ueberschufs dieses Reagens durch längeres Erhitzen auf $250^{\circ}$ entfernt.

Das nicht gewaschene Product orgab bei der Analyse Schwefel 9,71 pC. Es verlor beim Auswaschen $\mathrm{Na}_{2} \mathrm{SO}_{4}$, berechnet als Schwefel . 1,58 pC.

Schwefel . . . $\overline{8,13 \mathrm{pC} \text {. }}$

Demnach enthielt der auf diesem Umwege gewonnene blaue Körper nur wenig mehr Schwefel in chemischer Bindung, als das grüne Product, aus welchem er gewonnen war, nichtsdestoweniger hatten bei deın Erhitzen mit Schwefel 
zwei Vorgänge statt, nämlich eine Aufnahme derselbøn unter Bildung irgend welcher Verbindung, und cine Abgabe des näınlichen Körpers in der Form von Sulfat in nahezu gleicher Quantität.

2) Rohbrand erhitat mit $2 \frac{1}{2} p \mathrm{C} . \mathrm{Na}_{2} \mathrm{CO}_{8}$ und $\mathrm{C}$. Grünes Product. Das Filtrat, welches beim Auswaschen gewonnen wird, zeigt starke Reaction auf $\mathrm{Na}_{2} \mathrm{~S}$.

Analyse des gewaschenen und bei $100^{\circ}$ getrockneten Körpers :

$\begin{array}{lr} & 350 . \\ \mathrm{SiO}_{2} & 49,85 \\ \mathrm{Al}_{2} \mathrm{O}_{4} & 20,35 \\ \mathrm{~B} & 10,38 .\end{array}$

3) Rohbrand erhitzt mit $5 p \mathrm{pC} . \mathrm{Na}_{9} \mathrm{CO}_{3}$ und $\mathrm{C}$. - Der Tiegelinhalt ist heller grün. theilweise röthlich melirt. Beim Auswaschen giebt das Filtrat mächtige Fällung mit $\mathbf{P b A c}_{2}$.

Analyse des gewaschenen und bei $100^{\prime \prime}$ getrockneten Productes :

$\begin{array}{lr}\mathrm{SiO}_{z} & 49,96 \\ \mathrm{Al}_{\mathrm{y}} \mathrm{O}_{8} & 20,34 \\ \mathrm{~s} & 9,87 .\end{array}$

Nach dem Abbrennen mit Schwefel, längerem Erhitzen auf $250^{\circ}$, konnte beim Auswaschen $\mathrm{Na}_{8} \mathrm{SO}_{4}$ gewonnen werden, dessen Menge ist

$$
\text { auggedrückt in Schwefel } 1,62 \mathrm{pC} \text {. }
$$

Der gewaschene, bei $100^{\circ}$ getrocknete blaue Körper enthielt 9,98 pC. Schwefel.

Wir haben also auch in diesem Falle ähnliche Verbältnisse, wie sie unter 1) beschrieben wurden.

4) Rohbrand exhitzt mit $10 p \mathrm{C} . \mathrm{Na}_{2} \mathrm{CO}_{3}$. - Beim Auswaschen ergab das in Ganzen hellgrüne Product mächtige Fällung mit $\mathrm{PbAc}_{2}$ und enthielt nach dem Trocknen bei $100^{\circ}$ 8,72 pC. Schwefel. 
Diese grūnen Körper verhalten sich genau wie grũne Rohbrãnde, d. h. sie lassen sich bei jedem Erhitzen auf $100^{\circ}$ etwas Sulfat entziehen und verwandein sich wie diese im Laufe der Zeit in blaur Körper.

Bekanntlich erhielt Gentele beim Erhitzen des grünen Ultramarins mit Salmiak den blauen Farbstoff. Ich wiederholte diesen Versuch und fand ihn vollkommen bestätigt. Doch ist das nach dieser Methode erhaltene blaue Product von schlechtester Qualitat.

Erhitzt man in einer Kugelröhre, im Ofen oder künstlich bereitutes grünes Ultramarin mit Salmiak, so entweicht zunächst etwas Wasser und $\mathrm{H}_{2} \mathrm{~S}$, sodann $\mathrm{SO}_{2}$ und Schwefel, welchen letzteren man leicht mit sublimirtem Sulmiak an den külteren Stellen der Röhre verdichten kanı. Der Rückstand ist ein unschöncr, blan gefärbter Körper. Dieser wurde durch lang andauerndes stärkeres Erhitzen von allcin Salmiak befreit, ausgewaschen und das Filtrat untersucht. In diesem fuden sich Chlornatrium und Sulfat.

Ganz dieselben Erscheinungen nimnt man übrigens beim Erhitzen von Salmiak mit unterschwefligsaurem Natrium wahr. Es werden nämlich auch hier ziemlich bedeutende Mengen Schwefel gebildet, so dafs es scheint, dafs die Bläunng des grünen Uitramarins nur darauf heruhe, dafs der durch Zersetzung eines Componenten, sagen wir $\mathrm{Na}_{2} \mathrm{~S}_{2} \mathrm{O}_{3}$, freiwerdende Schwefel, unter Bildung von $\mathrm{Na}_{2} \mathrm{~S}_{5}$ resp. dessen Verbindung mit Thonerdenatronsilicat blau färbe.

Setzt man reines, ausgewaschenes, ständig feucht gehaltenes Ultramarin mehrstündiger (durch Spiegel verstärkter) Sonnenbestrahlung aus, so sind weder in dem entweichenden Wasserdampf, noch in dem Rückstand die geringsten Spuren eingetretener Oxydation nachweisbar. 


\section{Einige Betrachtungen.}

Soweit mir bekannt hat man bisher immer an der Annahme Pestgehalten, das Blauverden des Ultramarins beruhe auf der oxydirunden Wirkung des Sauerstoffs.

Wie früher erwähnt, findet man in gerissenen Tiegeln und an den Rändern der blauen Masse hâufig weifse Producte, welche beim Auswaschen mit Wasser (beinahe) ihren ganzen Schwefel in Form von Sulfat abgeben. Dafs diese Erscheinung in der That durch den Sauerstoff hervorgerufen wird, bedarf meines Erachtens keines weiteren Beweises. Hierbei geht jedoch die Farbe verloren, sie wird durch die oxydirende Wirkung zerstört. Ich glaube, dafs bei dem ganzen Bildungsprocesse des Ultramarins, als auch bei anderen Operationen, welche mit demselben vorgenommen werden, dem Sauerstoff keine andere Wirkung zukommt und stelle mir den ersteren in folgender Weise vor.

Der Salz sei richtig gewählt, fein gemahlen, innig gemischt und fest in den Tiegel geprefst, so dafs sich Theilchen bei Theilchen befindet. Der Ofen werde gelieizt.

Durch die Temperaturerhöhung wird der Schwelel, welcher sich an hande des Tiegels befiudet, zuerst erwärmt, er wird theilweise verflüchtigt, theilweise wirkt er auf die Soda ein. Es werden die Schwefelnatriumverbindungen und die Natronsalze der Schwefeloxydverbindungen gebildet. Ideal nach der Gleichung :

$$
3 \mathrm{Na}_{8} \mathrm{O}+12 \mathrm{~S}=2 \mathrm{Na}_{8} \mathrm{~S}_{6}+\mathrm{Na}_{8} \mathrm{~S}_{8} \mathrm{O}_{3} .
$$

Die schmelzenden Schwefelverbindungen werden vom Thone absorbirt. Mit steigender Temperatur gewinnt die Reaction an Lebhaftigkeit, sie dringt nach der Mitte des Tiegels vor, veranlafst dort gleichzeitig die Bildung von Umsetzungsproducten und das Entweichen von Schwefel, welcher jedoch nicht direct in den Ofenraum dringen kann, sondern 
gezwungen erst die äufseren Schichten passiren mufs und so gewissermafsen von Neuem Reactionen hervorruft, indem er z. B. $\mathrm{Nag}_{9} \mathrm{~S}$ in $\mathrm{Na}_{9} \mathrm{~S}_{5}$ urnwandelt. In diesem Sinne geht der Procers fort und wirkt z. B. die zugeschlagene Kohle als Reductionsmittel, sowohl der höheren Sauerstoffverbindungen des Schwefels, als auch des kohlensauren Natriums. Der entweichende Schwefel und das Kohlenoxyd verhindern den Sauerstoff an jeglicher Einwirkung.

Bei höherer Temperatur kommt der Thon (die etwa zuschlagene Kieselsäure je nach ihrem Zustande früher) zur Wirkung und zwar dergestalt, defs er die niederen Sauerstoffverbindungen ihres Natrons beraubt. Das so gebildete Thonerdenatronsilicat vereinigt sich molecular mit den gelösten Schwefelnatriumverbindungen. Und dieșe Verbindungen sind es nun, welche uns die analysirten Producte darstellen.

Das frühere geschilderte Verhalten des Ultramarins lehrt, dafs es selbst die Mittel in sich birgt, der oxydirenden Wirkung des Sauerstoffs lange zu widerstehen. Beim Abkühlen des Ofens entweicht fortwährend Schwefel, welcher bei der noch herrschenden hohen Temperatur durch sein Verbrennen den Sauerstoff so lange abhält, bis dieser keinen grofsen Schaden mehr veranstalten kann.

Der Abgang des Schwefels am Tiegelrande wird jedoch durch ständig neu zustrōmenden aus dem Innern orsetzt, so dafs gewissermafsen im Ofen selbst ein Röstprocefs erfolgt. Diese Annahme erklärt, wie mir scheint, ungezwungen die Bildung geschmolzener Kerne auch bei sonst gut gewāhltem Satze, indem sie diese Erscheinung auf den Mangel an Schwefel im Innern der Tiegel zurückführt, also den Einflufs schlechter Oefen und schlechter Tiegel in Betracht zieht und endlich noch ein Erklärungsmittel für die unconstante Zusammensetzung des Products an verschiedenen Orten eines und desselben Tiegels abgiebt. 
Der Annahme, das künstliche Ultramarin sei ein Gemengo durch Säure zerlegbarer Thonerdenatronsilicate, welche sich in Verbindung mit $\mathrm{Na}_{2} \mathrm{~S}_{5}, \mathrm{Na}_{2} \mathrm{~S}, \mathrm{Na}_{2} \mathrm{~S}_{2} \mathrm{O}_{3}$ befinden, steht meines Erachtens wenig im Wege.

Nach R ose zerfällt unterschwefligsaures Natron bei Luftabschlufs in Wasser, Schwefelwasserstoff und Schwefel, welche entweichen und in ein Gemenge von Schwefelmetall und schwefelsaures Salz, jedoch in je nach der Temperatur schwankenden Verhältnissen. - Das trockene Salz scheint diesem Zerfall selbst bei höherer Temperatur (Ofen) in Folge seiner feinen Yertheilung Widerstand entgegenzusetzen. Aufserdem läfst mir der ieichte Uebergang des $\mathrm{Na}_{2} \mathrm{~S}$ in $\mathrm{Na}_{2} \mathrm{~S}_{5}$ diese Vorstellung noch wahrscheinlicher erscheinen, so dafs meiner Meinung nach das ideale Ultramarin eine Verbindung eines Thonerdenatronsilicals mit Fünffuch-Schwefelnatrium ist.

Der Röstprorefs des Fabrikanten möchte daher nur ein Verfahren sein, das Product diesem idealen Ultramarin möglichst z.1 nähern. Wie uns der Rose'sche Satz lehrt, könnte selbst ein farbloses Thonerdenatronsilicat, welches sich in chemischer Verbindung mit unterschwefligsaurem Natron befindet (als Doppelsalz), bein Erhitzen zu einem blauen Körper werden.

Ich hoffe, dafs diese Arbeit, wenn sie auch nicht viel des Neuen bringt, dazu beitragen mag, die Ultramarinfrage ihrer Lösung näher zu bringen. 